\title{
PARAMETER ESTIMATION AND OPTIMIZATION OF THE HYDRONIC FLOOR HEATING SYSTEM AS A BASIS FOR Predictive ConTrol OF THE ZONE LEVEL ENERGY
}

\author{
Petar Maric, Ivan Bevanda \\ University of Mostar, Faculty of Mechanical Engineering, Computing and Electrical engineering, \\ Matice hrvatske bb, 88000, Mostar, BiH
}
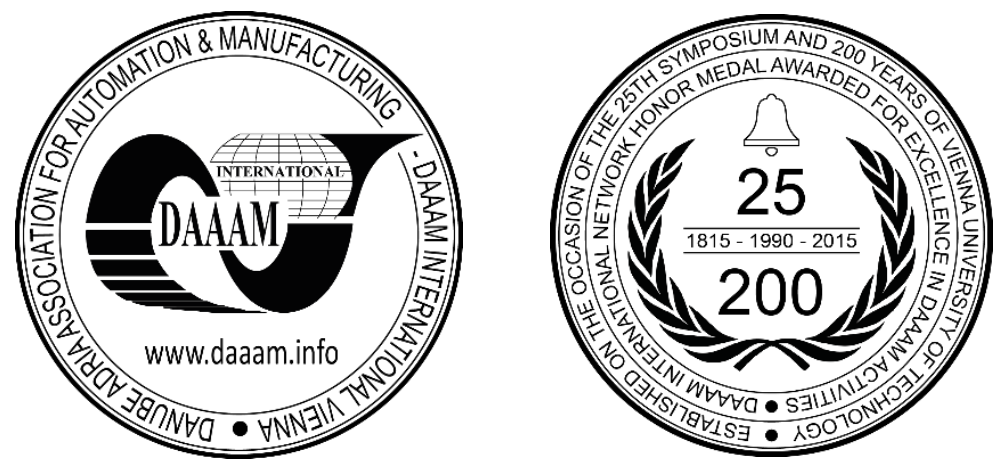

This Publication has to be referred as: Maric, P[etar] \& Bevanda, I[van] (2018). Parameter Estimation and Optimization of the Hydronic Floor Heating System as a Basis for Predictive Control of the Zone Level Energy, Proceedings of the 29th DAAAM International Symposium, pp.1038-1045, B. Katalinic (Ed.), Published by DAAAM International, ISBN 978-3-902734-20-4, ISSN 1726-9679, Vienna, Austria

DOI: $10.2507 / 29$ th.daaam.proceedings. 148

\begin{abstract}
Zone heating in residential objects has become one of the biggest energy spenders in the past few decades. Various strategies of the model predictive control have been assumed to be the best solution for the purpose of the smart distribution of the heating energy on the zone level in residential objects. In this paper the hydronic floor heating system has been examined in order to carry out the estimation and optimization procedure of the parameters related to the heat transfer. The zone model with the hydronic floor heating system has been developed and simulated with the help of simulation software IDA Indoor Climate and Energy (IDA-ICE) and the nonlinear differential first order model has been defined based on the inputs and outputs from the created IDA-ICE model. The nonlinear solver method has been used in order the carry out the optimization of the unknown parameters from the differential first order model. The optimization has been used for the calculation of the energy transmitted in to the zone and compared with the measured energy levels from the IDA-ICE simulation model.
\end{abstract}

Keywords: parameter estimation; hydronic floor heating system; nonlinear optimization; energy distribution; thermal energy control

\section{Introduction}

Efficient distribution of the heating energy in all types of objects occupied by the people has become one of the crucial environmental tasks in the 21.st century. Energy consumption in buildings today is regularly higher than the energy demands of the building alone. There are many possible reasons for the much higher energy consumption in the buildings: non rational energy management of the end users, non-optimal building design due to the insufficient applied insulation, old and inefficient energy distribution systems not adapted with the energy needs of the building. 
Approximately $40 \%$ of energy consumption worldwide is due to the energy management and energy distribution in the buildings through processes like heating, cooling and air conditioning systems [1]. Various studies and projects have been conducted through the world with the intention to assess and minimize the problem of the energy consumption in buildings. Some have shown that the energy savings greater than $11 \%$ per year in buildings are possible when the energy consumption in each zone was optimized [2]. Among these studies the model predictive control approach is recognized as a possible solution for the efficient energy management and distribution [3].

One of projects dealing with the problem of efficient energy management in the buildings is "Smart Building - Smart Grid - Smart City (3Smart)" project. Project is co-funded by the European Union through Interreg Danube Transnational Programme, and partners from the following countries are involved: Croatia, Slovenia, Austria, Hungary, Bosnia and Herzegovina and Serbia. The main objective of the 3Smart project is to provide a technological and legislative setup for cross-spanning energy management of buildings, grids and major city infrastructures in the Danube Region. It will provide optimal economical value to energy-efficiency and renewable energy investment in the building and at the same time it will result in optimized costs on the grid side whereas grid and buildings will also interact through exchanging energy and prices data [4].

This paper presents one part of the 3SMART project related to the zone level energy management. Hydronic floor heating/cooling system was mathematically described with the differential equations. These equations consist of a variables that were measured in the simulation software or on the pilot site and unknown parameters which describe the thermodynamic properties of the system. The simulation of the floor heating/cooling system has been conducted in the IDA ICE simulation software in a $4 m \times 4 m \times 3 m$ test room. During the simulation the temperature of the floor in the room was kept between $19{ }^{\circ} \mathrm{C}$ and $29^{\circ} \mathrm{C}$. Based on the simulation results, the unknown parameters which describe the thermodynamic properties of a system could be found using the nonlinear solver method.

The identified mathematical model of this floor heating/cooling system should later be used within the 3SMART project for the creation of the control algorithm of the heating energy distribution throughout the zone based on the energy reference supplied from the central MPC algorithm of the pilot building [3].

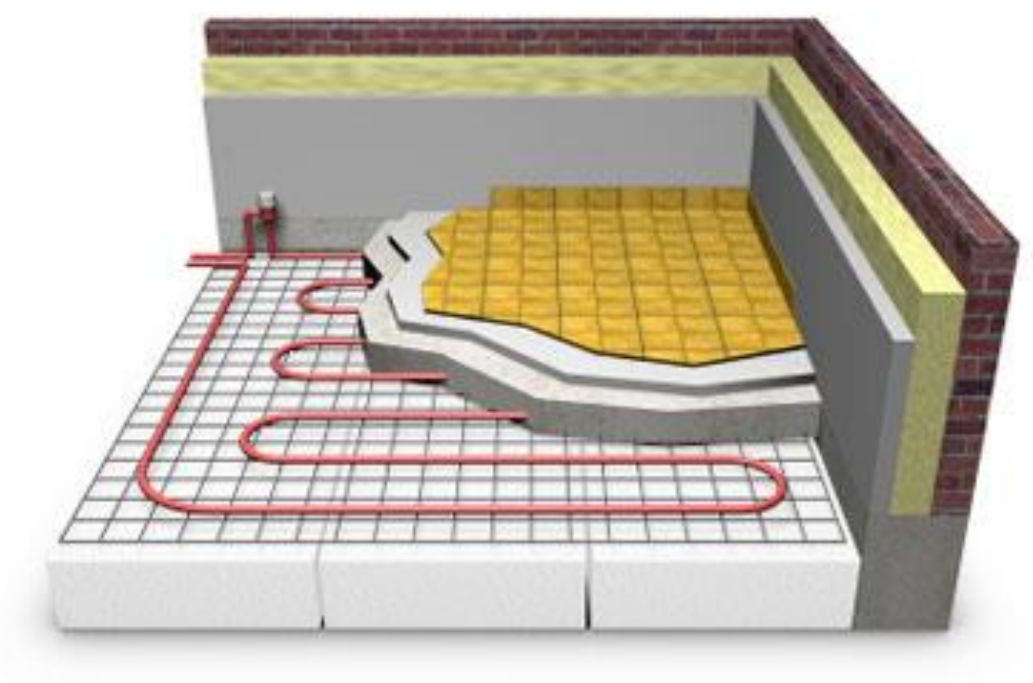

Fig. 1. Multilayer hydronic floor heating/cooling system[5]

A floor heating/cooling system of energy distribution achieves thermal comfort through convection, conduction and radiation. It has been widely used in the buildings because of its uniform temperature distribution. One another important advantage is that it can use low energy resources such as solar hot water, condensing boilers and heat pumps. In the design and the setup of a floor heating/cooling system, surface temperature is one of the most important variables to be considered [6]. In order to avoid the condensation, surface temperature should always be higher than the surrounding air temperature at a dew point. Based on this conclusion, the surface temperature should be kept between $19{ }^{\circ} \mathrm{C}$ and $29^{\circ} \mathrm{C}[7]$.

\section{Thermodynamic floor heating/cooling unit model}

For the purpose of the subsequent developments it is assumed that the water pipes are placed above the insulation layer. Concrete layer is then placed above the water pipes and finally a tile surface layer is applied on the top. Schematic view of the multilayer floor heating/cooling system can be seen on a Fig. 2. Thermodynamic mathematical model of a system is written and reviewed from the side of the outgoing water temperature circulating through the pipes. As previously stated, the heat transfer within the system occurs through the convection, conduction and radiation. First the heat is convected from the water circulating in the pipe to the inner wall of the pipe. After that the heat is conducted through the pipe material, concrete layer and the surface layer. The final stage of the heat transfer in to the zone is through the convection and radiation from the surface layer to the zone interior. 


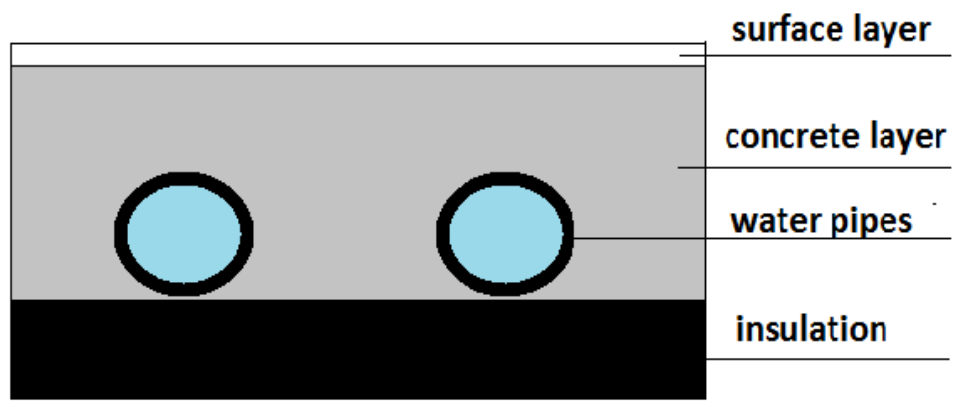

Fig. 2. Cross section of a floor view with the pipes immersed in to the concrete

The differential equation for the temperature of the returning water in the pipes $T_{w}^{o u t}$ can be written in the following form [8] [9] [10]:

$$
m_{w} c_{w} \frac{d T_{w}^{\text {out }}}{d t}= \begin{cases}Q_{w} c_{w}\left(T_{w}^{\text {in }}-T_{w}^{\text {out }}\right)-U\left(T_{c}-T_{z}\right)^{n} & Q_{w} \neq 0, \\ U\left(T_{c}-T_{z}\right)^{n} & Q_{w}=0\end{cases}
$$

where $m_{w}$ and $Q_{w}$ are respectively mass of the water inside the pipes and the medium mass flow of the water through the pipes. Parameter $c_{w}$ represents the specific heat capacity of the water. $T_{w}^{i n}$ stands for the temperature of the water going in to the pipes, $T_{c}$ stands for the temperature of the concrete layer above the pipes and the $T_{z}$ stands for the temperature of the air in the zone (room). The exponent $n$ can be set according to literature around 1 butt for the purposes of this research it is going to be treated as an unknown parameter that is going to be estimated with the use of nonlinear solver method.

Looking to the equation (1) from the energy perspective, one can see that the first part of the right hand side represents the energy inserted in to the system (concrete layer) whereas the second part represents the amount of energy transmitted from the concrete layer to the air in the room (zone). In that perspective the parameter $U$ stands for the heat transfer coefficient between the concrete layer and the air in the zone.

On the other side, looking from the thermodynamic perspective of the floor heating/cooling system one can surely notice due to the physical properties of a system the temperature of the outgoing water $T_{w}^{\text {out }}$ is never going to be greater than the temperature of the concrete layer $T_{c}$. More or less, these two are going to be very similar. Based on this assumption we can rewrite the equation (1) in the following form:

$$
m_{w} c_{w} \frac{d T_{w}^{\text {out }}}{d t}= \begin{cases}Q_{w} c_{w}\left(T_{w}^{\text {in }}-T_{w}^{\text {out }}\right)-U\left(T_{w}^{\text {out }}-T_{z}\right)^{n} & Q_{w} \neq 0, \\ U\left(T_{w}^{\text {out }}-T_{z}\right)^{n} & Q_{w}=0,\end{cases}
$$

From mathematical and identification standpoint, the equation (2) is much less complicated for the identification procedure than the equation (1). The main reason for the previous assumption and replacement is the constraint on the pilot site building of the 3SMART project where the temperture of the concrete $T_{c}$ is not going to be measured. Variables $Q_{w}, T_{w}^{\text {in }}, T_{w}^{\text {out }}$ and $T_{z}$ are going to be measured on the pilot site of the 3SMART project.

Now we can write the final differential equation of our model with parameters which are going to be estimated:

$$
\frac{d T_{w}^{\text {out }}}{d t}=a Q_{w}\left(T_{w}^{\text {in }}-T_{w}^{\text {out }}\right)-b\left(T_{w}^{\text {out }}-T_{z}\right)^{n}
$$

where $a$ parameter replaces the relation $1 / m_{w}$ and parameter $b$ replaces the relation $U / m_{w} c_{w}$. Three unknown parameters $a, b$ and $n$ will later be optimized and estimated with the help of MATLAB tools.

\section{Model simulation in IDA ICE software}

The floor heating/cooling system model has been developed in IDA ICE software for the puproses of the parameter identification and estimation. One can surely notice that the heat losses in the mathematical model equations have been neglected so the simulated zone (room) has been created in a similar manner with minimal losses to the environment. The dimensions of a zone construced in IDA ICE were $4 m \times 4 m \times 3 m$. The installed floor heating/cooling system had a maximum heating power of $100 \mathrm{~W} / \mathrm{m}^{2}$ with the pipes immersed in to the concrete. The thickness of a concrete layer in the floor was set up to $10 \mathrm{~cm}$ and the pipes were placed in the depth of $5 \mathrm{~cm}$. Above the concrete layer, the floor coating layer approximately $1 \mathrm{~cm}$ thick has been placed. 
The outputs of the floor heating/cooling system simulation were temperature of the outgoing water $T_{w}^{o u t}$ and the temperature of the air $T_{z}$ in the zone. The inputs to the simulation were temperature of the water going in to the pipes $T_{w}^{i n}$ and the medium mass flow of the water $Q_{w}$ through the pipes. These inputs were adjusted according to the figures (3) and (4). The simulation has been carried out for almost 120000 samples with the sampling time of 1 minute.

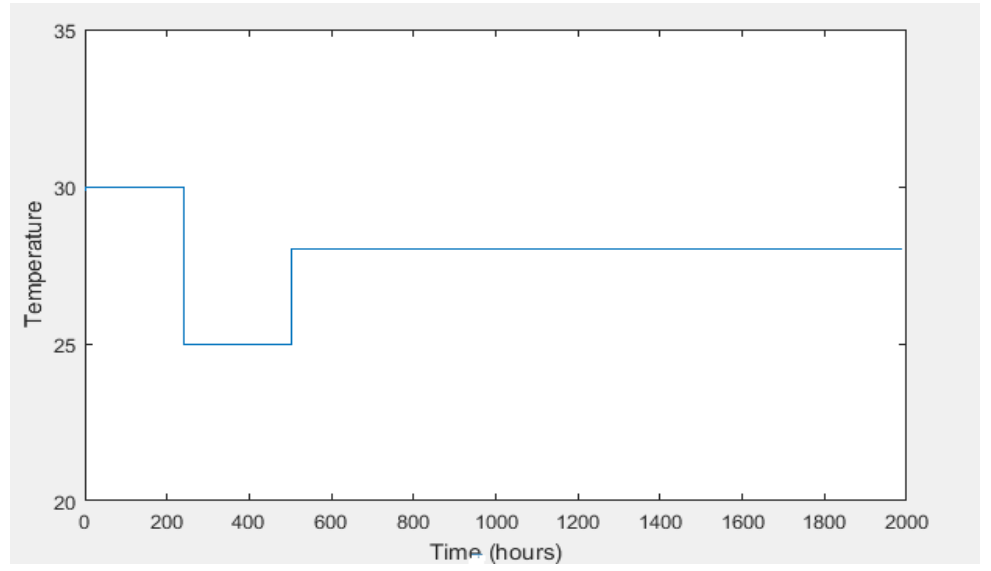

Fig. 3. Temperature of the water $T_{w}^{\text {in }}$ going in to the pipes

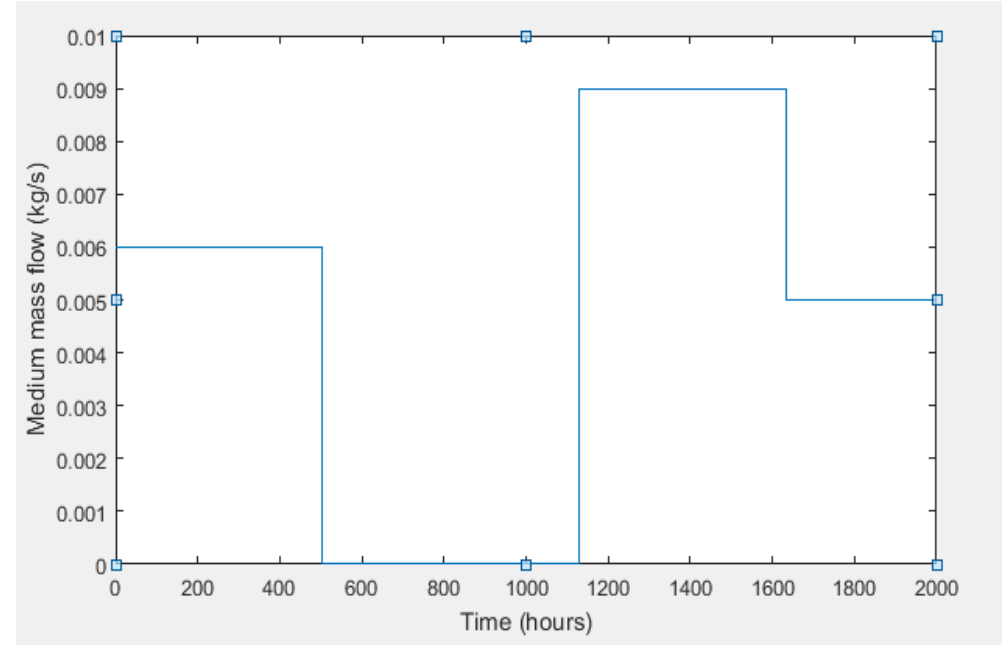

Fig. 4. Medium mass flow $Q_{w}$ through the pipes

The simulation outputs $T_{w}^{\text {out }}$ and $T_{z}$ are shown on the figures (5) and (6).

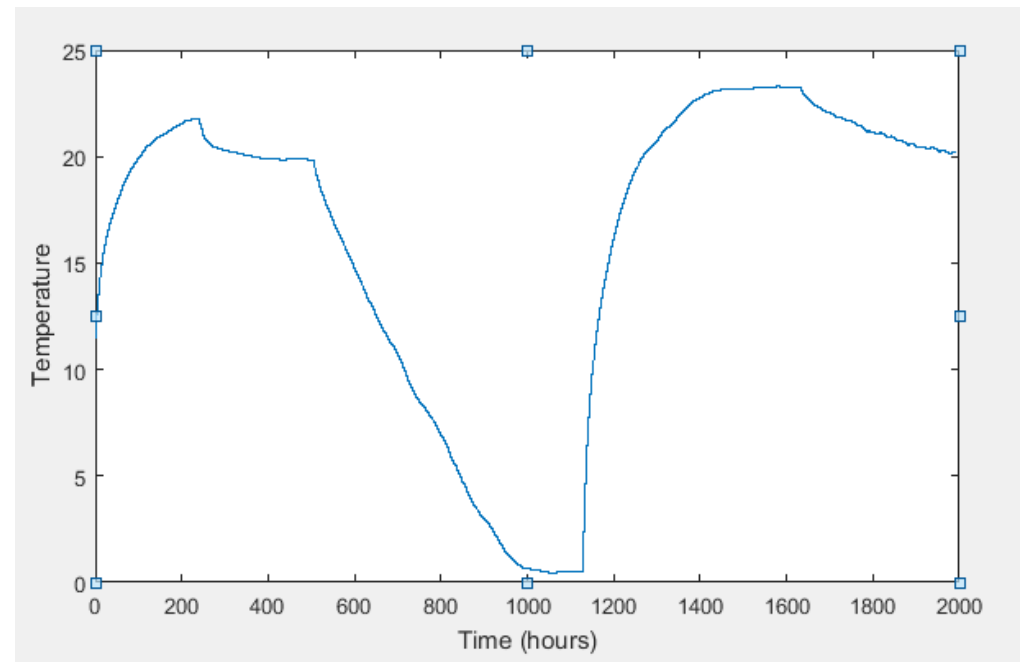

Fig. 5. Mean air temperature $T_{z}$ in the zone 


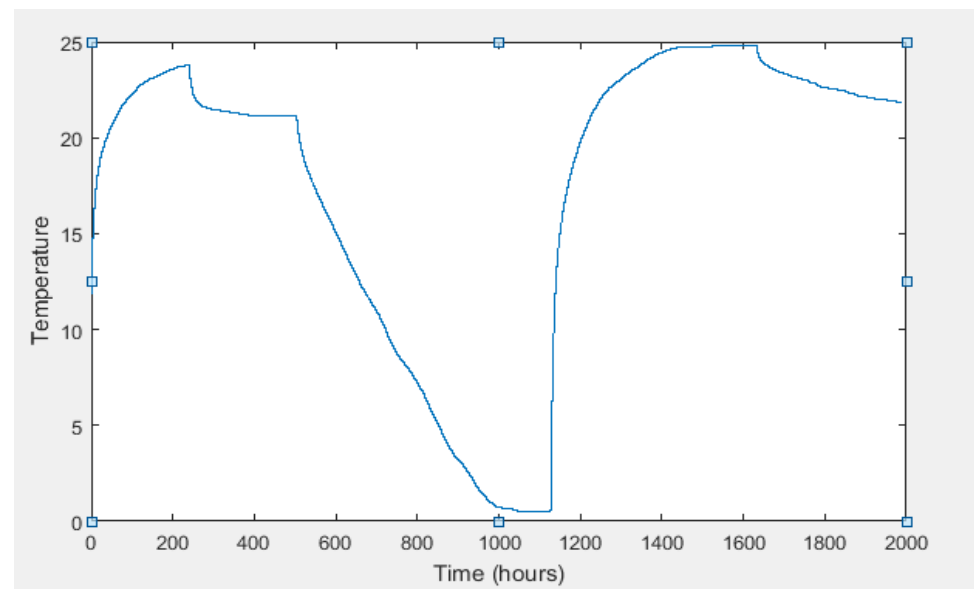

Fig. 6. Temperature of the outgoing water $T_{w}^{\text {out }}$ from the pipes

\section{Thermodynamic model parameter estimation}

In order to estimate the unknown parameters $a, b$ and $n$ from the equation (3) the nonlinear optimization procedure in MATLAB environment had to be applied based on the simulated data from the IDA ICE software simulation.

$$
\min _{\mathrm{a}, n, b}\left\|T_{\text {measured }}-T_{\text {sim }}\right\|^{2} .
$$

The equation (4) calculates the minimized squared error between the measured temperature of the outgoing water from the IDA ICE simulation and the simulated temperature of the outgoing water in MATLAB environment. Time response of the simulated model outgoing water temperature with respect to the measured temperature in IDA ICE can be seen on the figure (7).

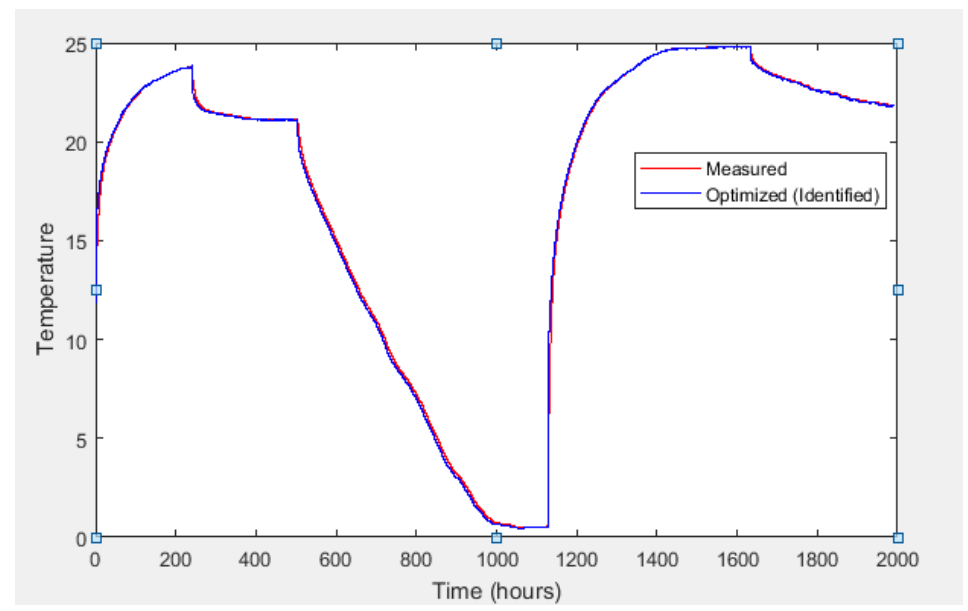

Fig. 7. Comparison between the measured temperature (IDA ICE) and the optimized temperature (MATLAB) of the outgoing water

It can be seen that the measured time response of the outgoing water temperature (IDA ICE) represents trully well the one optimized with the help of MATLAB nonlinear solver. This nonlinear optimization problem has been solved with the MATLAB-s -fminsearch and -fmincon commands giving the estimated values of $a, b$ and $n$ parameters: $b=0.0250$, $n=1.055$ and $a=1.25$. As it can be seen in the equation (3), the parameter $U$ cannot be found alone with this optimization procedure because of his connection and dependency with the denominator $m_{w} c_{w}$.

The estimation of the parameter $U$ was crucial for the completion of the estimation procedure for the floor heating/cooling system unit because of the importance of second part of the right hand side of the equation (2). This second part of the equation (2) should later be used to control the heat flow in to the zone based on the energy reference given by the central MPC in the building.

In order the fix the problem of finding the important unknown parameter $U$ (heat transfer coefficient) needed for the calculation of the energy transmitted in to the zone, the steady state points of energy transmitted from the water to the concrete were used. As previously stated the energy transmitted from the water to the concrete is defined by the first part of the right hand side of the equation (2). 
The points of steady state were found when the energy imported in to the system (concrete layer with the pipes) was equal to the energy transmitted from the system on to the air in the zone. The graphical presentations of the energy transmitted from the water to the concrete and of the energy transmitted from the concrete layer to the air in the zone can be seen on the figure (8) and figure (9).

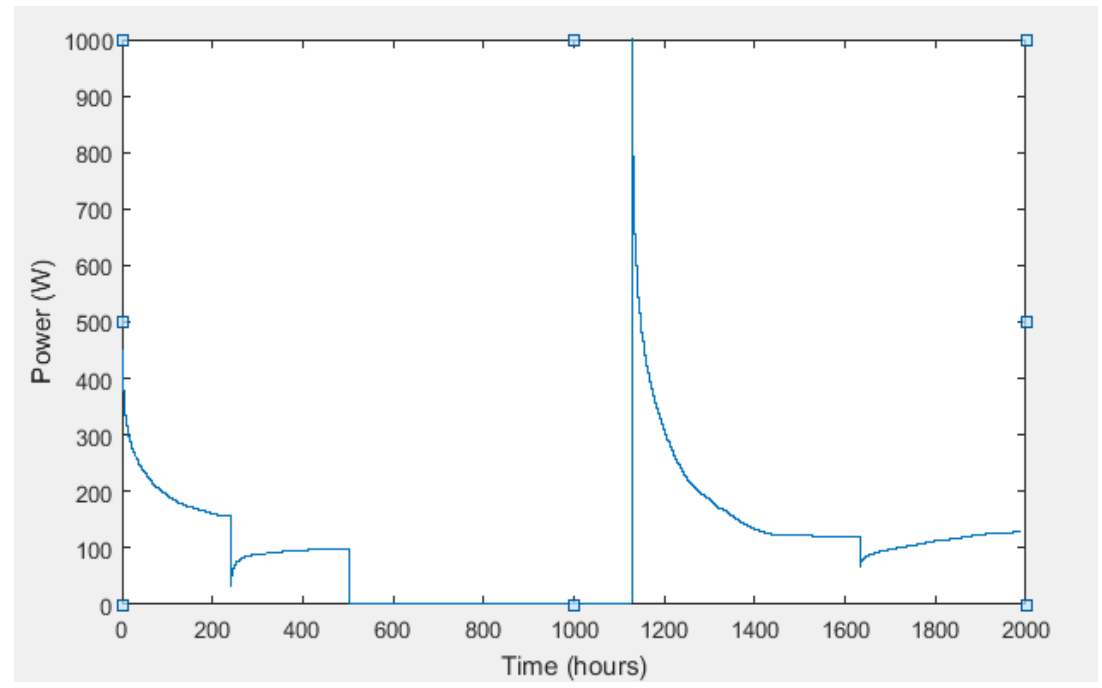

Fig. 8. Measured energy transmitted from the water on to the concrete layer

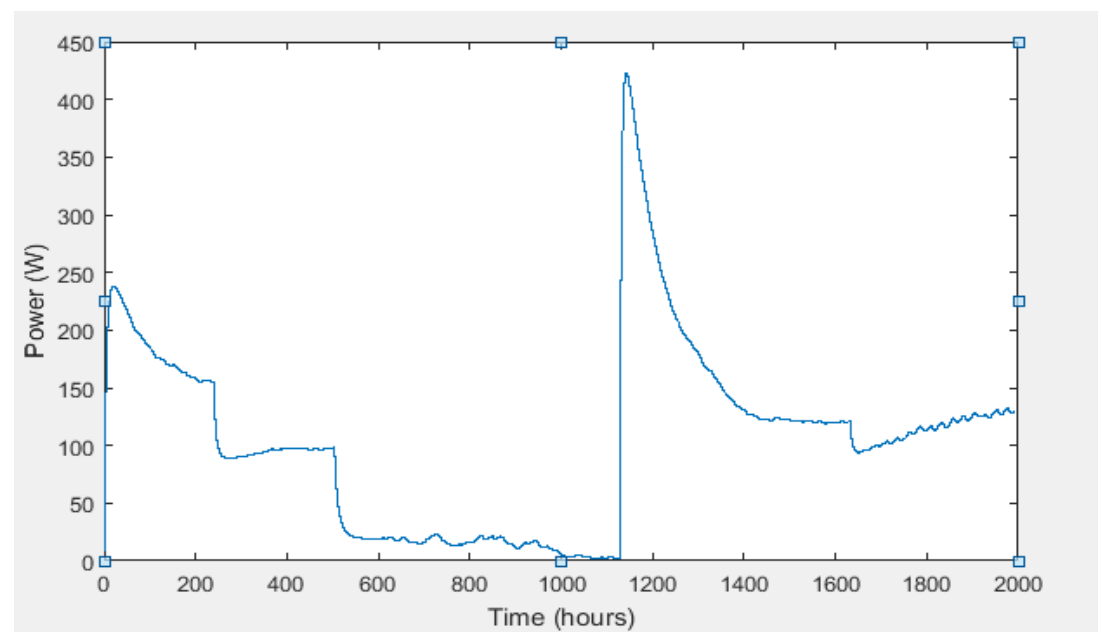

Fig. 9. Measured energy transmitted from the concrete layer on to the air in the zone

From the figures (8) and (9) four steady state points of energy have been pinpointed in order to calculate the value of heat transfer coefficient $U$ for the each steady state.

$$
U=\frac{P_{S S}}{\left(T_{w}^{\text {out }}-T_{z}\right)^{n}}
$$

where $P_{S S}$ represents the energy values in each of the four steady states. With the help of the equation (5) the average heat transfer coefficient $U$ was calculated with the calculated value of $U=75.5 \mathrm{~W} / \mathrm{K}$. Everything needed for the calculation of the thermal energy imported in to the zone by the zone actuators was estimated and found.

$$
\int_{0}^{T d} U\left(T_{w}^{o u t}-T_{a i r}\right)^{n} d t
$$

With the optimized parameter $n=1.055$ and heat transfer coefficient $U=75.5 \mathrm{~W} / \mathrm{K}$ we were able to calculate the energy transmitted in to the zone empirically and compare it to the measured energy transmitted in to the zone from the IDA ICE simulation which is shown on the figure (10). 


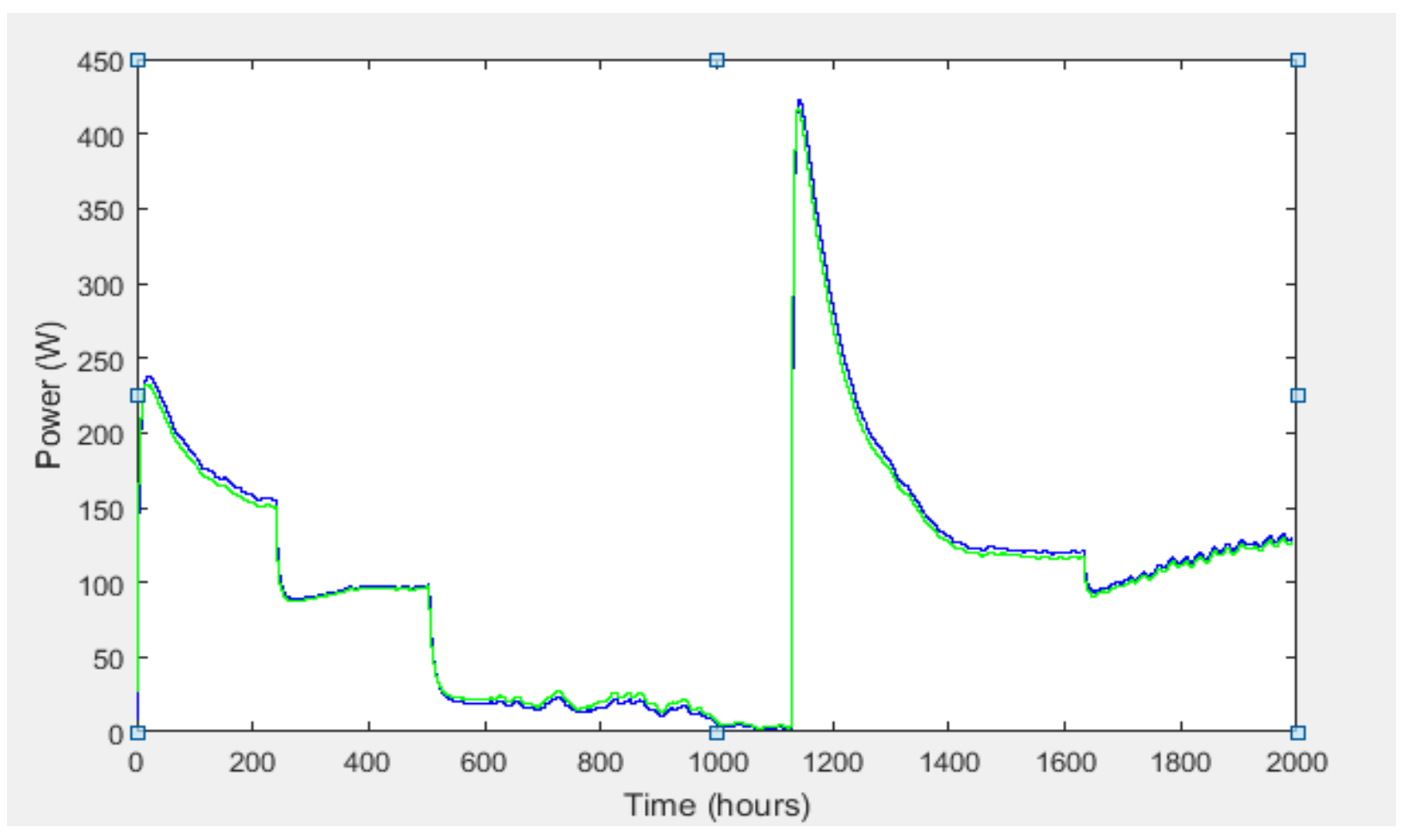

Fig. 10. Calulcated energy transitted to the air (green) compared with the measured energy - Transimtted to the air (blue) from IDA ICE software

As it can be seen the green line representing the energy calculated with the optimized parameters $n=1.055$ and $U=$ $75.5 \mathrm{~W} / \mathrm{K}$ truthfully follows the blue line which represents the energy measured from the simulation in IDA ICE software.

\section{Conclusion}

This paper represents the mathematical setup, optimization and identification procedure for the floor heating/cooling system. The floor heating/cooling system is a system that can easily be described with the ordinary differential equations that take in to account measurable variables such as temperatures of water going through the pipes, temeprature of the concrete layer, temperature of the air layer in the zone, medium mass flow of the water through the pipes and the physical properties of the system alone.

Because of the technical constraints of the 3SMART project this paper bases itself by describing the floor heating/cooling system without taking in to account the measurement of the concrete temperature and by writting the differential equation which depends on the temperature change of the outgoing water temperature. This mathematical setup is due to the technical implementation of the 3SMART project on the pilot building where the data about the concrete temperature is not going to be measured.

Later the identification of the system was reduced to the identification of the 3 separate parameters $a, b$ and $n$. These parameters had to be found with the nonlinear solver methods using MATLAB software environment. For that purpose the simulation in IDA ICE software has been carried out in order to simulate the floor heating/cooling system in one zone with the inputs and outputs representing the measured variables described in the differential equation. Because of the limitations of the nonlinear solver method and the dependence of the heat transfer coefficinet $U$ with the other physical parameters of the system the additional procedure had to be done so that the heat transfer coefficient $U$ could be found. The parameter $U$ has been calculated by the fact that the energies from the water side and from the zone (air) side are equal in a steady state.

This parameter is going to be crucial in future research of the control of the thermal energy inserted in to the zone by actuating the zone actuators connected to the medium mass flow of the floor heating/cooling system. As a results of this research the graphical comparison has been given, comparing the energy transmitted in to the zone in the simulation and the calculated energy transmitted in to the system with the opimized parameters $U$ and $n$.

One of the biggest limitations of this research is the inability to compare the calculated thermal energy transmitted in to the zone with the real data from the pilot building in the 3SMART project. These measured data from the pilot building are probably going to be available for the comparison and further research with the control of the thermal energy inserted in to the zone. 


\section{References}

[1] J. Birkeland, Design for sustainability: a sourcebook of integrated, eco-logical solutions. Earthscan Publications, 2002.

[2] B. Crnokic, G. Ljesic, Z. Stojkic, and M. Bandic Glavas, "Analysis of Energy Consumption in the Building as the Basis for Development of the Modular Model Predictive Control System," pp. 0299-0308, 2017.

[3] A. Martincevic, M. Vasak, and V. Lesic, "Model predictive control for energy-saving and comfortable temperature control in buildings," in 2016 24th Mediterranean Conference on Control and Automation (MED), 2016, pp. 298303.

[4] “Interreg Danube." [Online]. Available: http://www.interreg-danube.eu/approved-projects/3smart. [Accessed: 11Sep-2018].

[5] "Electric Radiant Floor Heating | HowStuffWorks." [Online]. Available: https://home.howstuffworks.com/homeimprovement/construction/materials/radiant-floor-heating2.htm. [Accessed: 11-Sep-2018].

[6] X. Jin, X. Zhang, and Y. Luo, "A calculation method for the floor surface temperature in radiant floor system," Energy Build., vol. 42, no. 10, pp. 1753-1758, Oct. 2010.

[7] B. Olesen, "Radiant floor heating in theory and practice," ASHRAE J., vol. 44, pp. 19-26, 2002.

[8] S. Provided, I. S. O. No, and I. H. S. Licensee, "Determination of the design heating and cooling capacity," in Building environment design- Design, dimensioning, installation and control of embedded radiant heating and cooling systems, International Organiation for Standardization, 2012, pp. 3-7.

[9] D. Roncati, "Iterative calculation of the heat transfer coefficient, Lisa finite element software analysis site," no. 2, 2013.

[10] D. Zhang, N. Cai, and Z. Wang, "Experimental and numerical analysis of lightweight radiant floor heating system," Energy Build., vol. 61, pp. 260-266, Jun. 2013. 